\title{
Depressão pós-parto em mulheres que tiveram cesárea não programada
}

\author{
Postnatal depression in Women who had unscheduled cesarean sections
}

Depresión postnatal en Mujeres que tuvieron cesáreas no programadas

Marília Medeiros de Matos ${ }^{1 *}$, Marina Déda Peixoto Leite ${ }^{2}$, Micaellem Rodrigues Santos da Silva ${ }^{3}$, Milena Gontijo Lemos Silva ${ }^{4}$, Natália Rafaela Olivotti ${ }^{5}$, Rayra de Sousa Sobral ${ }^{6}$, Roberta Flausino de Ávila7, Tainá Gomes Brandão ${ }^{8}$, Vivianne Araujo Rocha ${ }^{9}$, Anna Carla Garcia Cabral ${ }^{10}$.

\section{RESUMO}

Objetivo: Analisar os sintomas e as causas da depressão pós-parto (DPP), incluindo a cesárea não programada como um dos fatores de risco, bem como avaliar as consequências da depressão para a vida da mãe e do bebê. Revisão bibliográfica: Os sintomas da DPP costumam se manifestar entre a quarta e a oitava semana após o parto, devido às alterações hormonais, como os níveis aumentados de cortisol e as alterações da quantidade de estrogênio e progesterona que ocorrem durante a gravidez, associada a histórico familiar, fatores financeiros e psicossociais, muitas mães apresentam apatia, insônia, desesperança, sentimento de culpa, desejo a morte, dentre outros sintomas. Ademais, existe uma forte associação com a realização de cesáreas não programadas feitas quando a via normalmente apresenta risco à mulher ou ao bebê. Considerações finais: Nota-se que a DPP é um problema de saúde pública que gera um forte impacto na vida familiar, sendo assim, a importância de explorar as possíveis etiologias e incluir a cesárea de emergência como uma delas, leva ao desenvolvimento de ações que promovam um parto humanizado.

Palavras-chave: Cesárea, Depressão pós-parto, Gestante.

\begin{abstract}
Objective: To analyze the symptoms and causes of postpartum depression (PPD), including an unplanned cesarean section as one of the risk factors, as well as to evaluate the consequences of depression for the life of the mother and baby. Bibliographic review: The symptoms of PPD usually manifest between the fourth and eighth week after delivery, due to hormonal changes, such as increased levels of cortisol and changes in the amount of estrogen and progesterone that occur during pregnancy, associated with a family history, financial and psychosocial factors, many mothers have apathy, insomnia, hopelessness, guilt, desire for death, among other symptoms. In addition, there is a strong association with the performance of unplanned cesarean sections performed when the route normally presents a risk to the woman or the baby. Final considerations: It is noted that PPD is a public health problem that generates a strong impact on family life, therefore, the importance of exploring as possible etiologies and including an emergency cesarean as one of them, leads to the development of actions that promote a humanized delivery.
\end{abstract}

Keywords: Cesarean, Postpartum depression, Pregnant woman.

${ }^{1}$ Centro Universitário Unifacisa (UNIFACISA), Campina Grande - PB,

*E-mail: mariliamedeirosmatos@gmail.com

2 Universidade Tiradentes (UNIT), Aracaju - SE.

3 Universidade Federal de Goiás (UFG), Goiânia - GO.

${ }^{4}$ Faculdade Atenas Sete Lagoas (UniAtenas), Sete Lagoas - MG

${ }^{5}$ Centro Universitário Lusíada (UNILUS), Santos - SP.

6 Universidade Potiguar (UnP), Natal - RN.

${ }^{7}$ Faculdade Ceres (FACERES), São José do Rio Preto - SP.

${ }^{8}$ Faculdade de Ciências Médicas e da Saúde de Juiz de Fora (SUPREMA), Juiz de Fora - MG.

${ }^{9}$ Centro Universitário Tocantinense Presidente Antônio Carlos (UNITPAC), Araguaína - TO.

10 Universidade Católica de Brasília, Brasília - DF. 


\section{RESUMEN}

Objetivo: Analizar los síntomas y las causas de la depresión posparto (DPP), incluyendo las cesáreas no planificadas como uno de los factores de riesgo, así como evaluar las consecuencias de la depresión en la vida de la madre y del bebé. Revisión bibliográfica: Los síntomas de la DPP suelen manifestarse entre la cuarta y la octava semana después del parto debido a los cambios hormonales como el aumento de los niveles de cortisol y los cambios en la cantidad de estrógenos y progesterona que se producen durante el embarazo, asociados a los antecedentes familiares, a los factores económicos y psicosociales, muchas madres presentan apatía, insomnio, desesperanza, sentimientos de culpa, deseos de morir, entre otros síntomas. Además, existe una fuerte asociación con las cesáreas no programadas que se realizan cuando la vía normal presenta riesgos para la mujer o el bebé. Consideraciones finales: Se constata que la DPP es un problema de salud pública que tiene un fuerte impacto en la vida familiar, por lo que la importancia de explorar las posibles etiologías e incluir las cesáreas de urgencia como una de ellas conduce al desarrollo de acciones que promuevan un parto humanizado.

Palabras clave: Cesárea, Depresión posparto, Mujeres embarazadas.

\section{INTRODUÇÃO}

Em todo o mundo, estima-se que a Depressão pós-parto (DPP) esteja maciçamente presente em torno de 5 a $20 \%$ das mulheres, em contrapartida, especificamente no Brasil essa prevalência é elevada para 12 a $37 \%$ de diagnósticos confirmados (ARAÚJO IDS, et al., 2019). Assim, a DPP está incorporada nos impasses da saúde pública que afetam a saúde da mãe, a sua interação com seu filho e o desenvolvimento do mesmo. Com sua manifestação iniciando entre a quarta e a oitava semana pós-parto, esse quadro clínico de tristeza é intensificado progressivamente nos posteriores seis meses, incapacitando a mãe de realizar suas atividades cotidianas e contribuindo para a presença de apatia com tudo que a envolve (MORAES IGS, et al., 2006).

Essas mulheres são invadidas por uma espécie de melancolia que não sabem explicar, queixam- se de tristeza e irritabilidade, tal sentimento aparece algumas semanas depois do parto, vai ficando cada vez mais intenso ao ponto de torná-las incapazes de exercer as mais simples tarefas do dia a dia. Seu organismo passou por grandes modificações hormonais nos últimos meses que podem justificar este quadro (MORAES IGS, et al., 2006).

Nesse sentido, inicia-se um período que se estende de 45 a 60 dias pós-parto, chamado de puerpério, que é reconhecido por sua fase de deficiência hormonal, visto que as mulheres durante a gestação estão submetidas a altas doses de hormônios reprodutivos, os quais sofrem mudanças abruptas durante a gravidez e imediatamente após o parto. Diante disso, dentre esses hormônios, podemos citar o estrogênio e a progesterona, que além de sua funcionalidade na manutenção da reprodução, também são necessários para a integralidade do processamento emocional, da cognição, da motivação, da excitação e da modulação dos neurocircuitos envolvidos em estados afetivos normais e anormais (STEWART DE, et al., 2019).

Ademais, é mister citar que a maternidade é marcada não apenas por uma variação neuroquímica, assim, podemos acrescentar a essas mudanças as questões de caráter social, sexual e financeiro. Portanto, a combinação desses fatores no puerpério faz com que essa fase seja de grande vulnerabilidade para 0 aparecimento de desordens psiquiátricas (ARAÚJO IS, et al., 2019).

A depressão pós-parto, como supracitado, irá influenciar no estado afetivo da mãe e no comportamento e desenvolvimento da criança, sendo esse quadro um risco evidente em casos de depressão anteparto, depressão ou ansiedade durante a gestação, vida estressante, condições sociais e econômicas precárias, ausência de suporte e relações conjugais conflitivas (LÓPEZ-SÁNCHEZ I, et al., 2019).

Uma vez diagnosticada, a mulher apresenta humor deprimido, perda de interesse, anedonia, distúrbios de sono e apetite, concentração prejudicada, sentimento de culpa e inutilidade, pensamentos suicidas e preocupações obsessivas (STEWART DE, et al., 2019). Tal sintomatologia e o aparecimento da doença está associado ao modo de parto, sendo necessário incluir o pré-natal psicológico, visando a diminuição da prevalência de casos de DPP e a promoção da humanização da gestação, de acordo com Almeida NMDC e Arraiais ADR, et al. (2016). 
Correlacionado ao tipo de parto, a DPP está intimamente associada ao parto cesárea, tendo em vista que este último pode ser amplamente classificado dependendo do tipo da organização do processo, ou seja, se houve ou não, o planejamento. Diante disso, em casos de cesáreas não programadas, isto é, emergenciais, o foco é sempre livrar a mãe, o feto ou ambas de serem acometidos por alguma intercorrência e seus efeitos para a saúde maternal, fetal ou placentária (por exemplo: ruptura uterina, sofrimento fetal, eclâmpsia). Em contrapartida, ao mesmo tempo que esse tipo de parto busca oferecer a prevenção e os cuidados daqueles que estão envolvidos na gestação, também oferece riscos para à saúde física e psicológica da mulher, podendo sofrer consequências durante longos períodos (BENTON M, et al., 2019).

Portanto, diante do exposto, que ratifica a correlação da depressão pós-parto em mulheres que tiveram cesárea não programada, o presente artigo tem como objetivo analisar as causas e consequências desses dois fatores que assolam à saúde física e mental de diversas mulheres todos os anos.

\section{REVISÃO BIBLIOGRÁFICA}

\section{Sinais clínicos da DPP}

A gravidez é um período em que a mulher apresenta uma enorme vulnerabilidade para desenvolvimento de alterações do humor. Isso se deve ao fato das modificações que ocorrem em seu corpo e em sua rotina que acabam refletindo no estado emocional da mulher (ALOISE SR, et al., 2019). Contudo, é no período pósparto em que se vê a instalação da Depressão pós-parto (DPP), podendo estar ligada ao pré-parto, puerpério imediato, primeiros dias de vida do bebê, e até se estender para além do primeiro ano do mesmo (POLES MM, et al., 2018).

A DPP refere-se a uma série de sinais e sintomas que incluem, por exemplo, alterações cognitivas, psicomotoras e de humor que geralmente se manifestam entre a quarta e a oitava semana após o parto. Este período é marcado por várias mudanças hormonais, sociais e emocionais na vida da puérpera (ARAÚJO IS, et al., 2019). A DPP tem um diagnóstico considerado complexo devido aos diversos sintomas que a puérpera apresenta, os quais são colocados em segundo plano ou até mesmo justificado como apenas alterações hormonais decorrentes do parto (ARAÚJO IS, et al., 2019).

De acordo com o Manual de Ginecologia e Obstetrícia da Associação de Ginecologistas e Obstetras de Minas Gerais (SOGIMIG), alguns dos sintomas para classificar e diagnosticar a DPP estão ligados ao humor deprimido, tristeza, desânimo, choro fácil, ansiedade, alterações do apetite ou peso, insônia ou hipersonia, desesperança, baixa autoestima, sentimento de culpa, agitação ou lentidão, fadiga, dificuldade para tomar decisões, dificuldade de concentração, pensamentos contínuos de suicídio ou de morte. Além dos sintomas conhecidos como quadros depressivos, a puérpera com depressão pós-parto pode apresentar preocupação exacerbada com seu bebê ou pode apresentar também o extremo oposto, negligenciando os cuidados com o filho (SOGIMIG, 2012).

Entretanto, estes sintomas podem ser avaliados pela Escala de Depressão Pós-parto de Edimburg (EDPS), a qual é composta por dez itens que são assinalados pela própria gestante como forma de autoavaliação. Estes itens se referem aos sinais e sintomas mais frequentes que são observados durante a gestação ou no período puerperal (MALLOY-DINIZ LF, et al., 2009).

Um estudo realizado em uma maternidade brasileira na cidade de Salvador, utilizando a EPDS aliada a um questionário, conseguiu avaliar o perfil clínico das mulheres com a suspeita da depressão pós-parto e apontou que as características predominantes das mulheres com DPP são: idade de 24 anos, solteiras ou em união estável, baixa renda, pouca escolaridade, gravidez não planejada e complicações com o filho recémnascido. Esse estudo também indicou que as grávidas de baixa renda estão mais susceptíveis a desenvolver uma DPP, assim como de apresentarem um diagnóstico adequado e prosseguir para o tratamento. $O$ estudo indicou ser essencial disseminar os sinais e sintomas da depressão pós-parto, visto que, pode ser diagnosticado prontamente e encaminhado a um tratamento apropriado (ARAÚJO IS, et al., 2019).

Depois do parto, várias mudanças ocorrem no corpo, no cotidiano, nas despesas, enfim, na vida da mulher que podem influenciar no estado emocional de forma positiva ou negativa. Durante esse período, é importante 
que a puérpera receba um suporte adequado para conseguir assimilar tudo o que está ocorrendo. Neste momento, a maior parte delas se sente insegura, despreparada e vulnerável (POLES MM, et al., 2018).

A maternidade é um marco importante na vida de uma mulher, assim, o auxílio e suporte adequado durante esta fase pode evitar uma DPP. É importante a contribuição de todos os membros da família, pois quando o recém-nascido começa a habitar a casa e fazer parte da família, a rotina muda completamente. Esta mudança também ocorre no contexto da mulher, que quando se torna mãe, passa a ter alguém precisando exclusivamente de todos os cuidados e atenção, sendo que esta função passa a ser realizada na maior parte das vezes apenas pela puérpera (SOGIMIG, 2012).

Devido a essa dependência exclusiva, a mãe se encontra vulnerável, pois precisa manter seus próprios cuidados além dos cuidados do bebê e na maioria das vezes, precisa realizar também as tarefas domésticas. Conciliar uma carga de responsabilidade muito grande e encontrar tempo apenas para ela diante de tantas mudanças no seu cotidiano vai se tornando cada vez mais difícil, contribuindo para o esgotamento físico e emocional, que resulta em um sentimento de exaustão relatado por muitas mães (SOGIMIG, 2012).

A presença da família neste momento é de extrema importância para que a puérpera não se encontre nesse estado de exaustão, pois ela fica vulnerável para entrar em um quadro de DPP. É importante que a puérpera se sinta confortável, entenda que não está sozinha para cuidar do recém-nascido e que receba o apoio de alguém do seu círculo afetivo (BRASIL, 2005).

Tal suporte deve ser feito desde o início, quando se tem o diagnóstico de gravidez e nas consultas prénatal. Convidar algum membro da família sendo o pai do bebê ou outra pessoa do seu convívio, para auxiliar nas tarefas pode ajudar a manter um vínculo e trazer segurança para a gestante. Neste momento, é importante lembrá-la sempre de que ela não está sozinha e que poderá contar com o auxílio de outras pessoas. Os cuidados com a puérpera e o RN nas primeiras consultas durante o puerpério também são fundamentais para avaliar a saúde materna e do seu filho e classificar risco de DPP (BRASIL, 2005).

\section{Fatores de risco para a DPP}

No que concerne à etiologia da DPP, ainda não há uma delimitação concreta sobre o tema. Contudo, sabese que existe a atuação de múltiplos fatores que contribuem para o aparecimento de depressão puerperal, sendo eles: biológicos, psicossociais e sociodemográficos (LÓPEZ-SÁNCHEZ I, et al., 2019).

Os fatores biológicos são observados no eixo hipotálamo-hipófise-adrenal que aumenta os níveis de cortisol, o qual é responsável por preparar a gestante para o momento de estresse do parto. Após o nascimento do bebê, a puérpera apresenta quedas drásticas do cortisol, o que justifica a alteração de humor presente no puerpério. Tal alteração biológica é apontada como um dos fatores que levam ao desenvolvimento da DPP, porém as mudanças dos níveis hormonais são acompanhadas de outros fatores predisponentes (MONTENEGRO AC, 2012).

Em níveis psicossociais, estudos sobre a DPP consideraram a história psiquiátrica, eventos estressantes e falta de rede de apoio como fatores que levam ao desenvolvimento do quadro. Nesse nível, o histórico pessoal e familiar de transtorno psiquiátrico foram pontos elencados nas entrevistas realizadas em parturientes em Salvador, sendo os principais fatores encontrados em parturientes com depressão puerperal. Ademais, os conflitos conjugais, a gravidez não planejada e a ausência de suporte durante o puerpério foram elementos que também estiveram presentes em puérperas com DPP (ARRAIS ADR, et al., 2018).

No que diz respeito aos níveis socioeconômicos, existe uma relação da baixa escolaridade e classe social com a manifestação da DPP. Essa relação se justifica pelo simples raciocínio de que a renda per capita pequena contribui para aparecimento de dificuldades financeiras que podem se correlacionar com eventos de estresse para a parturiente. Em vista disso, observa que os fatores de riscos para a DPP atuam interrelacionando e não se sobrepondo, ou seja, é um cenário cujo qual pode existir a somatização de vários fatores e em diversos níveis (MELO SB, et al., 2018).

Outros pontos que, também, teriam relação com a depressão puerperal seriam os fatores obstétricos que a parturiente vivenciou, podendo ser intercorrências de partos anteriores, perdas neonatais ou a necessidade de cesárea não eletiva (ECKERDAL P, et al., 2018). 


\section{Associação entre cesárea e a DPP}

A cesárea é uma técnica cirúrgica que tem como objetivo realizar uma incisão no abdômen e no útero da paciente para retirada do bebê. Como qualquer procedimento cirúrgico, ela tem vários pontos a serem avaliados como: (I) riscos, (II) benefícios, (III) indicações e (IV) a técnica a ser realizada, sendo de extrema importância avaliar e listar todas as complicações que podem ocorrer durante o procedimento e seus cuidados pré-operatórios (SOGIMIG, 2012).

No que concerne as indicações para cesárea, a literatura afirma as seguintes recomendações: as anomalias de apresentação (situação transversa, apresentações pélvicas), deformidades pélvicas, prolapso do cordão umbilical, HIV positivo de acordo com a avaliação da carga viral da gestante, infecção por vírus herpes simples, desproporção cefalopélvica, parada de progressão do trabalho de parto, placenta prévia, gravidez gemelar (SOGIMIG, 2012).

Segundo dados do Ministério da Saúde (MS), a ocorrência de cesáreas no Brasil em 2006 foi de aproximadamente $45,5 \%$, dados da Agência Nacional de Saúde (ANS) foram registrados de 55,6\%, no cenário mundial são registrados de $10 \%$ a $15 \%$ dados oferecidos pela Organização Mundial de Saúde (OMS). Em relação às complicações mais comuns na cesárea, podem ser citadas as hemorragias, lacerações uterinas, lesões do trato urinário, parada cardíaca, ventilação insuficiente (QUEIROZ RR, et al., 2019).

Na revisão de literatura que foi a base para a presente investigação, observou-se que o crescente número de partos por cesárea está associado a vários fatores, sendo eles socioculturais, financeiros, opção do médico e autonomia da gestante. Apesar do aumento das cesáreas realizadas, a OMS admite que não houve mudanças nas taxas de mortalidade materna e neonatal, sendo que a cesárea tem como indicação para ser realizada na urgência e para promover benefícios durante o trabalho de parto (ARRAIS AL, et al., 2018).

Em relação ao ponto de vista dos profissionais da área, para alguns médicos, a cesárea é vista como um processo rápido e que irá trazer mais benefícios e segurança para a mãe e o recém-nascido. Para outros, já deve ser indicada como foi citado anteriormente, pois pode oferecer mais riscos à puérpera além de contribuir para uma recuperação dolorosa e lenta. É importante salientar que com a implantação da Rede Cegonha do Ministério da Saúde em 2011, com objetivo de humanização do parto e boas práticas de atenção ao parto e ao nascimento, foi concedido maior autonomia a gestante e oferecê-la um suporte adequado, dessa forma auxiliando para a escolha de qual via poderá ter seu filho (QUEIROZ RR, et al., 2019).

Sobre a depressão pós-parto, objeto deste estudo, a literatura demonstra que estudos feitos para avaliar a associação da cesárea com a DPP tiveram impactos diferentes. A realização desse procedimento pode estar relacionada a um fator de risco que envolve a DPP principalmente cesáreas de emergência (ARRAIS AL, et al., 2018).

A partir da produção científica a respeito da DPP, encontraram fatores de riscos para pacientes desenvolverem depressão pós-parto das 76 mulheres que fizeram parte do estudo, 47 passaram pelo parto cesariano, associando também a 23 fatores de riscos diferentes que contribui ou tem relação com a DPP. Já o estudo realizado em Brasília (DF), no período de janeiro a abril de 2011, nos hospitais privados foi selecionado 107 mulheres gestantes para serem avaliadas, dessas 11 apresentavam sintomas depressivos durante a gravidez, mas que foram excluídas após o parto (ZACONETA AM, et al., 2013).

Foi observado que 12 mulheres tiveram sintomas depressivos pós-parto diante do que foi apresentado no estudo não pode concluir uma relação de mulheres que passaram pelo procedimento cirúrgico da cesárea com a depressão pós-parto. Neste sentido, o distúrbio pode ter surgido durante períodos variados dependendo da gestante podendo ser durante a gestação ou no período puerperal, mas a cesárea pode ter envolvimento para desenvolver e estar relacionada a um fator de risco (ZACONETA AM, et al., 2013).

\section{Consequências da DPP}

A DPP é um grande desafio para a saúde pública uma vez que promove consequências no funcionamento materno, no neurodesenvolvimento e no desempenho cognitivo dos recém-nascidos (ROJAS G, et al., 2018).

Em relação à mãe, os sintomas da DPP afetam os padrões biológicos que são importantes para a regulação das respostas fisiológicas do organismo. Dentre estes, o eixo hipotálamo-hipófise-adrenal é 
afetado, levando a um aumento dos níveis de cortisol, hormônio que modula as respostas do organismo frente às situações de estresse. Uma vez aumentado, o cortisol provoca sintomas psicológicos que se iniciam até mesmo durante a gravidez, mesmo que a depressão venha a ser estabelecida só após o nascimento do bebê. Dentre estes sintomas, que podem aparecer até mesmo no primeiro trimestre de gestação, podem ser ressaltados a somatização, ansiedade, estados depressivos, estresse (SÁNCHEZ TAPIA FR, et al., 2017).

Em relação a construção do vínculo afetivo entre mãe e filho, a DPP traz grande prejuízo, uma vez que a mãe em estado depressivo acaba não estabelecendo as primeiras interações com bebê o que interfere negativamente no desenvolvimento social, afetivo e cognitivo da criança (CUMMINGS, et al., 1994; DODGE, 1990; FIELD, 1998; TRONICK e WEINBERG, et al., 1997). A mãe, que deveria ser a figura que supre as necessidades do filho, acaba negligenciando os cuidados e não atende às necessidades específicas da faixa etária da criança (TOLENTINO EC, et al., 2016).

Alguns estudos mostram que as mães com DPP têm mais dificuldade de exercer as funções da maternidade, se sentem incompetentes e acabam não se sentindo conectadas emocionalmente aos seus bebês (MILGRON, et al., 1996). Esta falta de conexão se inicia nos primeiros contatos entre o binômio mãefilho. A mãe depressiva, ao direcionar o olhar ao bebê, rompe o contato e não o restabelece, gasta menos tempo tocando, falando e interagindo com o seu filho, apresentam mais expressões negativas do que positivas, são menos responsivas e espontâneas (SÁNCHEZ TAPIA FR, et al., 2017).

Como reflexo dessa falta de interação, os bebês de mães depressivas se mostram menos responsivos, têm uma aparência depressiva, semblante de tristeza e raiva, se irritam e protestam mais frequentemente, não mantém o contato visual, vocalizam menos e são menos ativos se comparados aos bebês de mães não depressivas (TOLENTINO EC, et al., 2016). Além disso, as mães deprimidas acabam apresentando menos brinquedos e prendendo menos a atenção de seus filhos. Quando isto ocorre no final do primeiro ano de vida, principalmente, resulta em um menor desenvolvimento cognitivo na criança quando esta atinge dezoito meses e aos quatro anos (SÁNCHEZ TAPIA FR, et al., 2017).

\section{CONSIDERAÇÕES FINAIS}

Por meio deste estudo, conclui-se que a depressão pós-parto se tornou um problema de saúde pública com comprometimento físico e psíquico à mãe e ao bebê. Nesse contexto, é possível compreender que a via de parto é considerada como um fator de risco para o desenvolvimento da DPP. De acordo com os dados utilizados nesse artigo, há um consenso, assegurando que, quando se analisa a via parto isoladamente, entende que este seja um importante fator de risco para esse distúrbio, concluindo que essa patologia possui uma etiologia multifatorial, é válido ressaltar que, as cesáreas de emergência, são consideradas um fator que predispõe a DPP, que comprova a necessidade de um pré-natal bom e humanizado.

\section{REFERÊNCIAS}

1. ALMEIDA NMC, ARRAIS AR. O Pré-Natal Psicológico como Programa de Prevenção à Depressão Pós-Parto. Psicologia: Ciência e Profissão, 2016; 36(4): 847-863.

2. ALOISE SR, et al. Depressão pós-parto: identificação de sinais, sintomas e fatores associados em maternidade de referência em Manaus. Enfermagem Foco, 2019; 10(3): 41-45.

3. ARAÚJO IS, et al. Postpartum Depression: Epidemiological Clinical Profile of Patients Attended In a Reference Public Maternity in Salvador-BA. Revista Brasileira de Ginecologia e Obstetrícia, 2019; 41(3): 155-163.

4. ARRAIS AR, et. al. Fatores de Risco e Proteção Associados à Depressão Pós-Parto no Pré-Natal Psicológico. Psicologia: Ciência e Profissão, 2018, 38(4): 711-729.

5. BENTON M, et al. Women's psychosocial outcomes following an emergency caesarean section: A systematic literature review. BMC pregnancy and childbirth, 2019; 19(1): 1-24.

6. BRASIL. Ministério da Saúde. Pré-natal e Puerpério: atenção qualificada e humanizada. Brasília: Ministério da Saúde, 2005; 163p.

7. CARTER FA, et al. Cesarean Section and Postpartum Depression: A Review of the Evidence Examining the Link. Psychosom Med., 2006; 68(2): 321-330.

8. ECKERDAL $P$, et al. Delineating the association between mode of delivery and postpartum depression symptoms: a longitudinal study. Acta Obstet Gynecol Scand, 2018; 97: 301-311. 
9. GONZÁLEZ-GONZÁLEZ A, et al. Factores asociados a depresión posparto. Revista Archivo Médico Camagüey, 2019; 23(6): 1025-0255.

10. LÓPEZ-SÁNCHEZ I, et al. Factores asociados a depresión posparto. Revista Archivo Médico de Camagüey, 2019; 23(6): 770-779.

11. MALLOY-DINIZ LF, et al. Escala de Depressão Pós-Parto de Edimburg: análise fatorial e desenvolvimento de uma versão de seis itens. Revista Brasileira de Psiquiatria, 2010; 32(3): 316-318.

12. MELO SB, et al. Sintomas depressivos em puérperas atendidas em Unidades de Saúde da Família. Revista Brasileira de Saúde Materno Infantil, 2018; 18(1): 171-177.

13. MONTENEGRO AC. Cortisol salivar e depressão pós-parto. Tese (Doutorado em Neuropsiquiatria e Ciência do Comportamento). Universidade Federal de Pernambuco, 2012; $101 \mathrm{p}$.

14. MORAES IGS, et al. Prevalência da depressão pós-parto e fatores associados. Revista de saúde pública, 2006; 40(1): 65-70.

15. POLES MM, et al. Sintomas depressivos maternos no puerpério imediato: fatores associados. Acta Paul Enfermagem, 2018; $31(4): 351-358$.

16. QUEIROZ RR, et al. Assistência prestada às mulheres que foram submetidas à cesariana por parada de progressão. REME - Rev Min Enferm, 2019; 23: e-1204.

17. REIS TL, et al. Autonomia feminina no processo de parto e nascimento: revisão integrativa da literatura. Rev Gaúcha Enferm. 2017; 38(1): e64677.

18. ROJAS G, et al. A Remote Collaborative Care Program for Patients with Depression Living in Rural Areas: Open-Label Trial. J Med Internet Res., 2018; 20(4): e158.

19. SÁNCHEZ-TAPIA FR, et al. Frecuencia de tendencias suicidas (suicidalidad) y factores asociados en mujeres durante el primer año posparto. Revista de Neuro-Psiquiatria, 2017; 80(3): 172.

20. SCHWENGBER DDS, PICCININI CA. O impacto da depressão pós-parto para a interação mãe-bebê. Estudos de Psicologia, 2003; 8(3): 403-411.

21. SOGIMIG. Associação dos Ginecologistas e Obstetras de Minas Gerais. Saúde mental da mulher na gravidez e no puerpério, Belo Horizonte: Coopmed, 2012; 1117p.

22. STEWART DE, VIGOD SN. Postpartum depression: pathophysiology, treatment, and emerging therapeutics. Annual Review of Medicine, 2019; 70: 183-196.

23. ZACONETA AM, et al. Depression with postpartum onset: a prospective cohort study in women undergoing elective cesarean section in Brasília, Brazil. Rev. Bras. Ginecol. Obstet., 2013; 35(3): 130-135. 